\title{
Dynamic Vibration Absorber for Squeal Noise Suppression in Simple Model Structures
}

\author{
Meifal Rusli*, Mulyadi Bur and Lovely Son \\ Mechanical Engineering Department, Faculty of Engineering \\ Andalas University, Kampus Unand Limau Manis \\ Padang 25163, Indonesia \\ *meifal@ft.unand.ac.id
}

Received 2 December 2013

Accepted 17 September 2014

Published 31 October 2014

\begin{abstract}
In recent research it was found that squeal noise caused by friction-induced vibration can result in mode coupling instability. Presently, there is no method that can be reliably used to eliminate this kind of noise. This paper is focused on the use of dynamic vibration absorbers (DVAs) to suppress the generation of squeal noise. The performance of the DVA is investigated numerically for two simple cases, i.e. a simple two-degree of freedom model, and an L-shape space frame. It is found that the DVA can be applied to shifting or reducing the unstable region of mode coupling, by which the unstable region is removed from the operating condition. Particularly, the addition of the DVA in horizontal direction on the near-point-of-friction can possibly avoid unstable mode coupling. However, the addition in vertical direction will increase the possibility of squeal noise incident. Moreover, a high frequency DVA in horizontal direction at the near-point-of-friction shifts the unstable region into higher normal contact stiffness and higher friction coefficient. Consequently, addition of a mass with very stiff spring or a rigid mass in the horizontal direction can prevent the occurrence of unstable mode coupling, as long as it is not coupled with the vertical direction. If the added mass affects the dynamic behaviors in both vertical and horizontal directions, squeal noise in the original normal contact stiffness can still occur.
\end{abstract}

Keywords: Squeal noise; mode coupling instability; dynamic vibration absorber; normal contact stiffness; friction-induced vibration and noise.

\section{Introduction}

Squeal noise is found frequently when one metal counter face slides over another metal counter face under certain conditions. In many observations, this kind of noise is generated within the frequency spectrum of $1 \mathrm{~Hz}$ to $20 \mathrm{kHz}$ dominated by a few sharp peaks, and often just a single frequency mode. The frequencies are found to

\footnotetext{
${ }^{*}$ Corresponding author.
} 
correspond closely to the natural frequencies of the structure. However, not all modes are affected by self-excitation due to this mechanism.

Two typical mechanical systems that are commonly found generating squeal noise are train wheels moving on a curved track ${ }^{1-4}$ and brake systems. ${ }^{5-8}$ Various empirical methods have been developed in order to reduce squeal noise for these cases. For example, Remington found that lubrication addition on the contact surface successfully can suppress squeal noise for some types of wheels, but is worthless for other types. ${ }^{9}$ Eadie attached a water-based-liquid material on the top of the rail, and showed that it can effectively decrease squeal noise level by about $10-15 \mathrm{dBA} .{ }^{10}$

Moreover, Brunel analyzed the implementation of a ring damping solution consisting of a machined semi-cylindrical groove under the wheel rim, where a metallic circular ring is inserted into this groove. This method can successfully reduce squeal noise from $5 \mathrm{~dB}$ to $10 \mathrm{~dB}$ in some wheels, but is ineffective in many others. ${ }^{11}$ Moreover, Botto utilized an active noise control to suppress the squeal noise in trains. The idea can control the noise propagation; however, it cannot overcome the squeal noise generation problem. ${ }^{12}$

Furthermore, in brake squeal suppression, Fosberry and Holubecki first introduced the application of radial slots in the brake shoes, circumferential slots between the rib and the web, axial slots in the rib, and a damper acting against the web of the drum, which were effective in suppressing squeal noise in some drum brakes. ${ }^{13}$ Hamabe suggested that addition of some masses into the backing plate of the drum brake can reduce squeal noise level. ${ }^{14}$ Also, the application of ultrahigh frequency excitation into an active control of disc brake squeal noise was investigated by Cunafare. ${ }^{15}$ However, the methods can only be applied to some brake configurations or to some automobile types but are ineffective to the others.

Meanwhile, tuned-mass damper (TMD) or dynamic vibration absorber (DVA), a device consisting of a mass, a spring, and a viscous or hysteretic damper was successfully used to reduce the vibration level of numerous structures due to harmonic excitation at a certain frequency (tonal tuning). Since it was proposed in 1909, the DVAs have been widely used in machineries, buildings, and structures. The first theoretical investigation of the DVA design can be found in the text by Den Hartog. ${ }^{16}$ By balancing two fixed points in the frequency response, the optimal tuning ratio and damping ratio can be obtained for an auxiliary single-degree-offreedom (SDOF) mass attached to an undamped system. Later, the DVA application to SDOF and MDOF systems with some inherent damping was then examined, and various tuning rules were proposed in the time and frequency domains including $\mathrm{H} 2$ and $\mathrm{H}$ optimal tunings. ${ }^{17}$

On the other case DVA application, Bakre investigated numerically the optimum parameters of multiple tuned mass dampers (MTMDs) for suppressing the dynamic response of a base-excited damped main system. It was observed that an optimal design of the MTMD system was more effective for vibration control than a single TMD. ${ }^{18}$ Also, for suppressing undesirable vibrations of a structure under 
underground accelerations, Khan investigated the effectiveness and robustness of multiple dual TMDs (DTMDs), consisting of several units of DTMDs. ${ }^{19}$

Furthermore, Miguelez investigated the behavior of boring bars with a passive DVA for chatter suppression by modeling the boring bar as a cantilever EulerBernoulli beam. ${ }^{20}$ The distribution of vibrational energy in members of a complex structure with tuned absorbers attached at the joints and subjected to dynamic loadings has been studied. ${ }^{21}$

In application of the DVA-like device to reduce squeal noise, Abu-Bakar proposed some models of drum brake for squeal noise suppression through structural modifications using the finite element method. One of the methods is the addition of mass to the drum. ${ }^{22}$ It was found that the added mass could eliminate a frequency peak of squeal noise, but with the others remaining unchanged.

In this paper, a basic application of the DVA to the squeal noise suppression is investigated. Recently, it was found that the squeal noise is generated in a mechanism of mode coupling instability caused by friction-induced vibration. ${ }^{23}$ In the squeal noise elimination, the DVAs are implemented to prevent mode coupling instability. The performance of the DVAs is investigated numerically for two simple cases: a TDOF model, ${ }^{24,25}$ and an L-shape space frame. ${ }^{25}$

\section{Simple Two-Degree-of-Freedom Model}

In order to investigate the performance of the DVA in suppression of squeal noise caused by mode coupling instability, a simple model of a single-mass TDOF system proposed by Hamabe, ${ }^{15}$ Hoffman, ${ }^{24}$ and modified by Rusli ${ }^{25,28}$ is attached by two spring-mass systems each in vertical and horizontal direction (Fig. 1). The particle mass $M$ is held by two linear springs $k_{1}$ and $k_{2}$, and other two linear springs $k_{n}$ and $k_{t}$, which represent a normal and a tangential contact stiffness between the particle mass and friction surface, respectively. The spring $k_{2}$ leans toward $45^{\circ}$ from the normal direction. The mass is in contact with a moving plane that has a constant velocity via a contact point $\mathrm{O}$. Also, $F_{n}$ and $F_{f}$ represent the normal and the friction force, respectively, acting on the contact plane at point $\mathrm{O}$. Then, two mass-spring

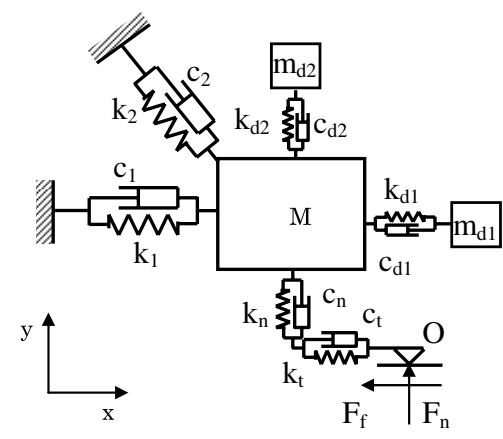

Fig. 1. TDOF model and double dynamic absorbers. 
dampers are attached to the particle mass in horizontal and vertical directions, of which the masses are $m_{d 1}$ and $m_{d 2}$ and the stiffnesses are $k_{d 1}$ and $k_{d 1}$. In this case, the spring constants $k_{1}$ and $k_{2}$ are kept constant, and viscous damping ratios are zero. The effects of the friction coefficient and the normal contact stiffness to the mechanism of squeal noise generation are taken into account and the performance of DVAs to cancel mode coupling instability is investigated.

In this section, numerical simulations are carried out to investigate the performance of the DVA in preventing mode coupling instability. The idea of using the double DVAs is inspired by the fact that the instability occurring within two vibration modes are coupled in the form of conjugate complex for the eigenvalues and eigenvectors. By assuming that the damping ratios are zero and the relative displacement of point $\mathrm{O}$ to the mass $M$ is negligible, the equations of motion of the particle mass can be formulated as follows:



Since the stiffness matrix in Eq. (1) is nonsymmetric, it is obvious that this system has complex eigenvalues in the form of pairs of conjugate eigenvalues and conjugate eigenvectors for certain values of matrix components. A pair of eigenvalues generates a mode coupling to originate instability at certain values of matrix components. ${ }^{26}$ If the stiffnesses of the structure $\left(k_{1}\right.$ and $\left.k_{2}\right)$ are constant, then mode coupling interaction will depend on the contact parameters, i.e. friction coefficient, normal contact stiffness, and tangential contact stiffness.

The free response of modal system is generally expressed by $q_{i}(t)=Q_{i} e^{(a+b j) t}$, where the imaginary part of the exponential power represents the natural frequency, and the real part represents the growth rate of the response. If the real part of the eigenvalue is positive, as found in the pair of conjugate eigenvalues, the vibration response will increase exponentially with time. In other words, the system is unstable.

The influence of structural damping on this kind of instability has not yet been fully clarified. Some researchers believe that high damping ratio can reduce the possibility of squeal noise generation, although there is no clear clarification about 
this point. Theoretically, under specific conditions, the mode coupling instability may be regarded as a viscous instability in the sense that an increase in structural damping may render a stable system unstable. ${ }^{27}$

In previous research, it was observed that the contact parameters; i.e. normal contact stiffness, tangential contact stiffness, and the friction coefficient about the sliding contact surface, play a significant role in generating unstable mode coupling in a structure with friction. ${ }^{28}$ This unstable mode coupling occurs within certain values of the contact stiffness and friction coefficient. Higher values of friction coefficient will raise the possibility of generation of mode coupling instability.

In the case of friction on a rough surface of sliding contact interface, the contact parameters depend on the topography characteristics of the contact surface as long as the load applied on the interface is not large enough to destroy all asperities. A rougher contact surface has lower contact stiffness than a smoother one. Because of the interaction between the dynamic parameters of the structure and the contact parameters in forming mode coupling, it can be understood why some structures generate squeal noise in relatively rough surface and other structures generate in smoother surface. ${ }^{28}$

Furthermore, there are only few models that can explain about the tangential stiffness of a sliding contact. The ratio between tangential and normal stiffness in a static sliding condition varies from 0.48 to 0.68 , depending on the values of parameters such as oscillation frequency, material, sliding speed, normal and tangential load. ${ }^{29}$ The ratio between dynamic and static tangential stiffness at very low frequency $(0-400 \mathrm{~Hz})$ is around $0.25-0.35 .^{28}$ Because of the lack of theory about the tangential contact stiffness in the higher frequency range, in this model, the tangential contact force that depends on the tangential contact stiffness is accumulated with the total friction force as a function of the total friction coefficient.

For the simplest model shown in Fig. 1 , we set $M=1 \mathrm{~kg}, k_{1}=k_{2}=1 \mathrm{~N} / \mathrm{m}$, and the friction coefficient at a constant value of 0.5 . The eigenvalues of this structure without DVA will change as a function of the normal contact stiffness as shown in Fig. 2. Clearly, two natural frequencies merge at a point for a certain value of contact
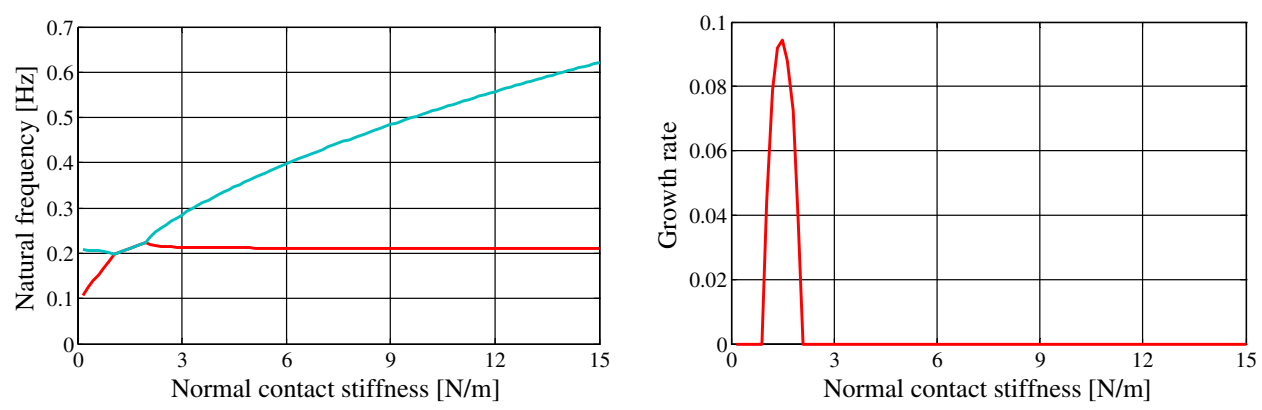

Fig. 2. Natural frequency and growth rate of TDOF model without DVA. 
stiffness (around $1 \mathrm{~N} / \mathrm{m}$ ), and separate again at a higher value (around $2 \mathrm{~N} / \mathrm{m}$ ). The merging natural frequency is about $0.2-0.22 \mathrm{~Hz}$. The growth rate is positive in the merging region; therefore, the vibration response of the structure grows into instability. This normal contact stiffness within $1-2 \mathrm{~N} / \mathrm{m}$ then is defined as the original normal contact stiffness that generates squeal noise in the next discussion.

In general, the DVA is applied to reducing undesirable vibrations by minimizing the vibration amplitude and energy level. Den Hartog found the optimal tuning ratio for an auxiliary SDOF mass attached to an undamped SDOF system as:

$$
f_{\mathrm{opt}}=\frac{1}{1+v},
$$

where $v$ is the ratio of the damper mass to the system mass. It means that a higher mass ratio will shift the optimal frequency to a wider tuning frequency ratio.

Now, the performance of the DVA for the TDOF system with the friction device in Fig. 1 is presented in Figs. 3 and 4. Figure 3(a) shows when the mass ratio is $v=0.05$ and the damper frequency $f$ is $0.2 \mathrm{~Hz}$ (close to the unstable mode coupling frequency in the original normal contact stiffness), the unstable mode coupling moves to a higher frequency with the normal contact stiffness falling within $1.2-2.4 \mathrm{~N} / \mathrm{m}$. It is observed that unstable vibration still occurs around the original normal contact stiffness without the DVA $(1-2 \mathrm{~N} / \mathrm{m})$. By increasing the mass ratio and frequency damper in the horizontal direction as shown in Figs. 3(a)-3(c), the performance of the DVA to suppress unstable mode coupling increases. Higher frequency damper shifts the unstable mode coupling to the higher normal contact stiffness. However, the increasing mass ratio does not play a significant role.

In contrast, the application of the DVA in vertical direction is ineffective to avoid the occurrence of unstable mode for all mass ratios and damper frequencies. Figure 4 shows the unstable mode coupling persistent in the original normal contact stiffness and in wider contact stiffness range $(1-4 \mathrm{~N} / \mathrm{m})$. It means that the DVA in vertical direction increases the possibility of squeal noise generation.

Figure 5 shows the stability diagram in terms of the normal contact stiffness and the friction coefficient. Unstable mode coupling with positive growth rate is represented by gray and white region. In the original condition without DVA, positive growth rate occurs when the friction coefficient is higher than 0.4 and the normal contact stiffness is within $1-3 \mathrm{~N} / \mathrm{m}$. When the vertical DVA is applied with various mass ratios and damper frequencies (Figs. 5(b)-5(d)), the gray region grows wider than the one with lower friction coefficients around the original normal contact stiffness. Hence, the utilization of the DVA in the vertical direction increases the possibility of occurrence of unstable modes.

When the horizontal DVA is applied with various mass ratios and damper frequencies (Figs. 6(a)-6(d)), the gray region is constricted by the increase of mass ratio $v$. The unstable vibration is avoided in the region with lower friction coefficients and original contact stiffness. However, another unstable region appears for higher normal contact stiffness. If the original normal contact stiffness remains unchanged, 



(a)
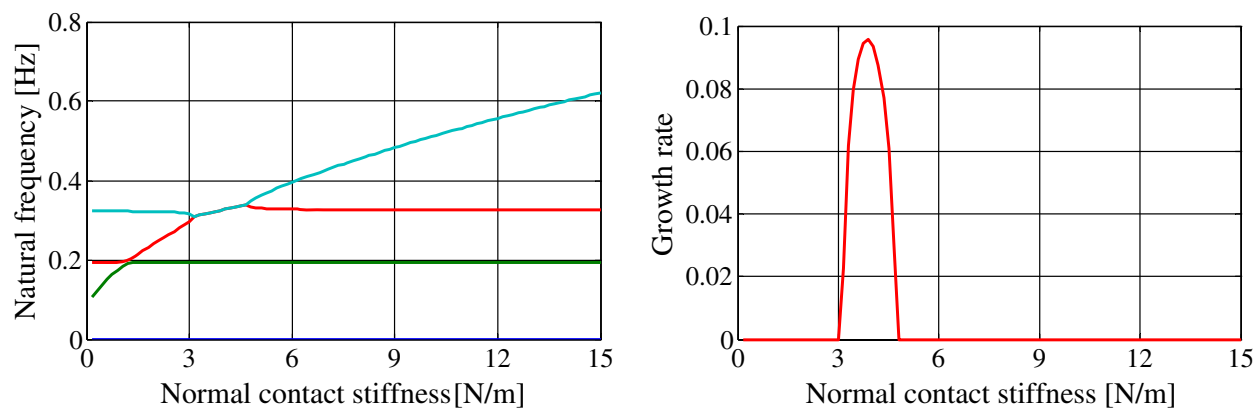

(b)
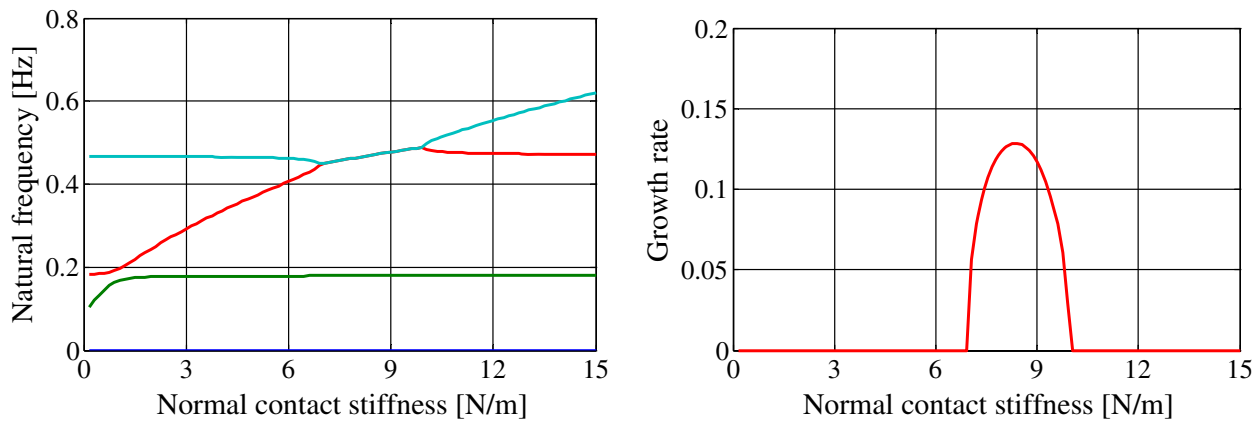

(c)

Fig. 3. Natural frequency and growth rate for DVA applied in horizontal direction with: (a) $v=0.05$ and $f=0.2 \mathrm{~Hz}$, (b) $v=0.2$ and $f=0.3 \mathrm{~Hz}$ and (c) $v=0.3$ and $f=0.4 \mathrm{~Hz}$.

squeal noise caused by unstable mode vibration will not exist. Therefore, higher frequency damper shifts the gray region to the region of higher contact stiffness.

Consequently, an added mass with a very stiff spring or a rigid mass in the horizontal direction will prevent the generation of unstable mode coupling as long as it is not coupled with the vertical one. If the added mass is involved in the vibration 



(a)
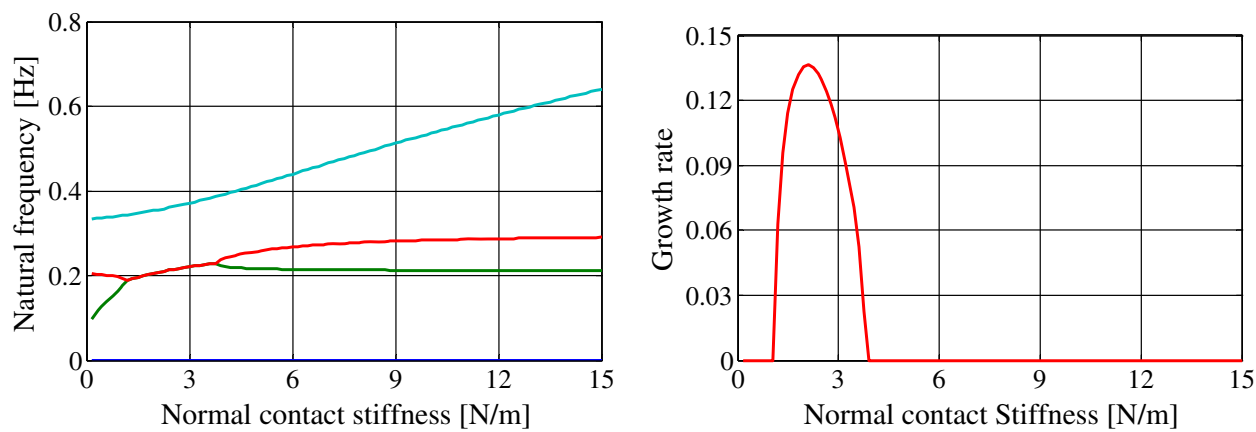

(b)
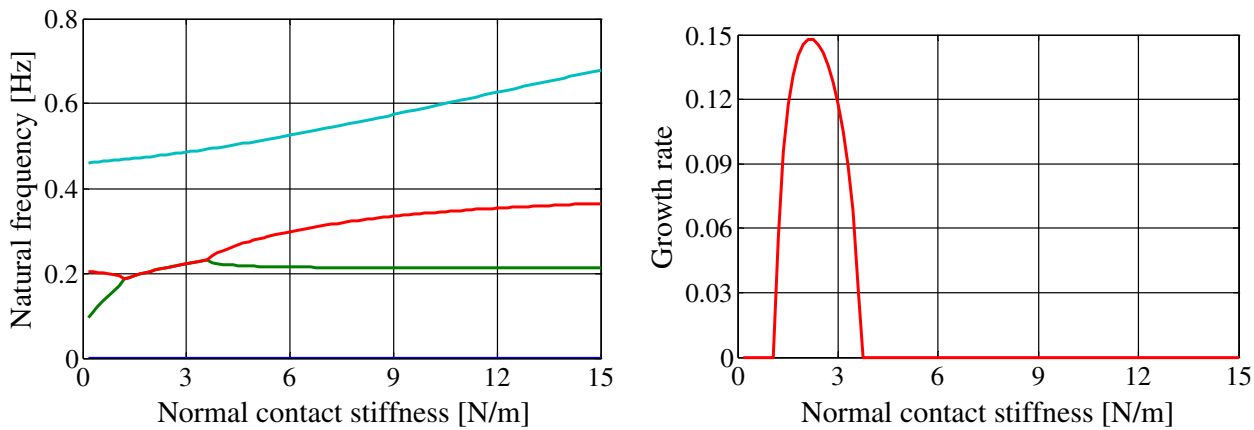

(c)

Fig. 4. Natural frequency and growth rate for DVA applied in vertical direction with: (a) $v=0.05$ and $f=0.2 \mathrm{~Hz}$, (b) $v=0.2$ and $f=0.3 \mathrm{~Hz}$ and (c) $v=0.3$ and $f=0.4 \mathrm{~Hz}$.

of both the vertical and horizontal direction, squeal noise with the original normal contact stiffness can still occur.

The frequency response function of the system using the state space analysis by Matlab with and without DVA is illustrated in Fig. 7, where the normal contact 


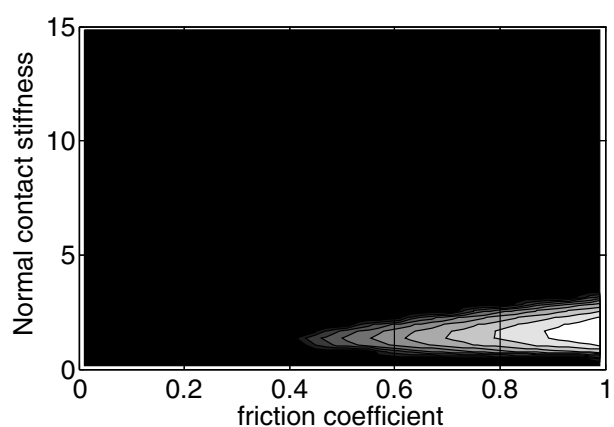

(a)

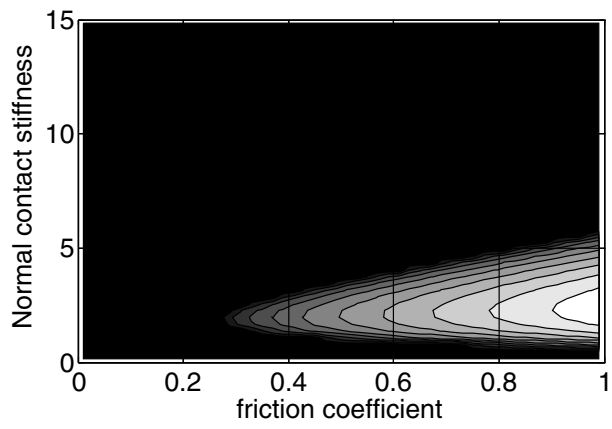

(c)

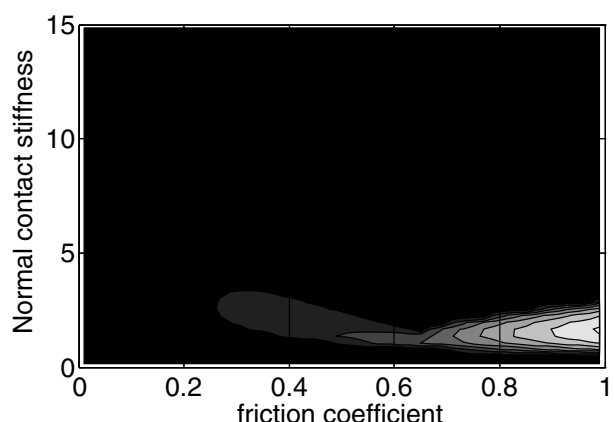

(b)

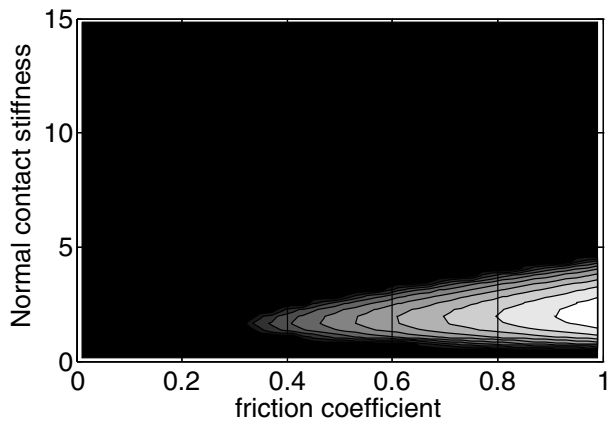

(d)

Fig. 5. Stability region of the system with DVA of: (a) $v=0$ (no DVA), (b) $v=0.05$ and $f=0.2 \mathrm{~Hz}$ in vertical direction, (c) $v=0.3$ and $f=0.4 \mathrm{~Hz}$ in vertical direction, (d) $v=0.2$ and $f=0.5 \mathrm{~Hz}$ in vertical direction.

stiffness is set $1.5 \mathrm{~N} / \mathrm{m}$ and the friction coefficient is assumed to be 0.5 . It is observed that the system without DVA has only a single peak around the frequency of $0.22 \mathrm{~Hz}$ and has lower amplitude than the models with DVAs. This seems to be quite odd in comparison with the eigenvalue analysis which clearly indicates that the model without DVA should have higher amplitude than that with one or two DVAs.

It is well known that a dynamical system has two types of responses when excited by external load; namely, the transient and the steady state responses. In a stable damped system, the steady state response or the frequency response has dominant amplitude compared with the transient one due to the fact that the transient response decreases logarithmically with time caused by the damper. Conversely, in unstable mode coupling that generates the squeal noise, the frequency response is very small and dominated by unstable transient response that logarithmically increases due to the positive real eigenvalue. Therefore, although the system without DVA has very low frequency response as shown in Fig. 7, since the transient response has positive growth rate, so the vibration grows exponentially. Surely, the added 


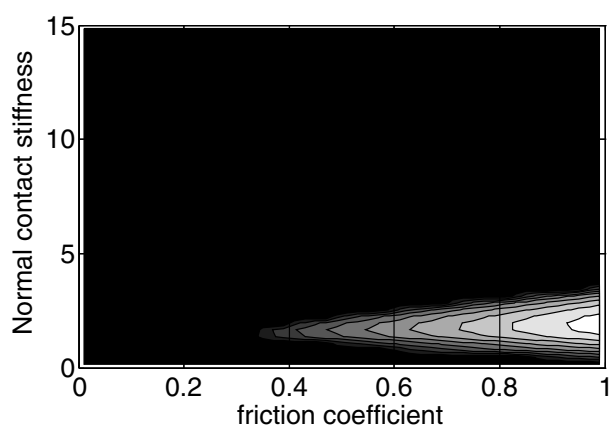

(a)

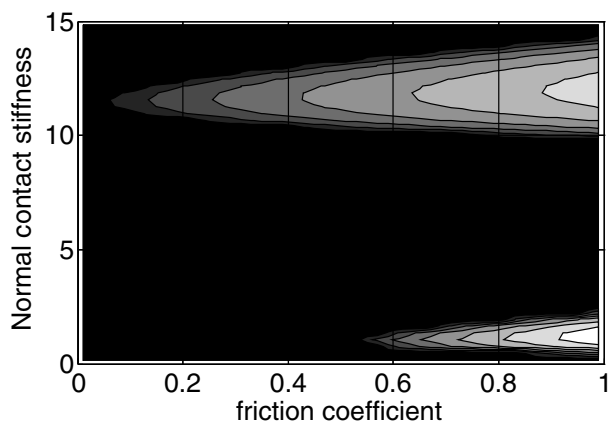

(c)

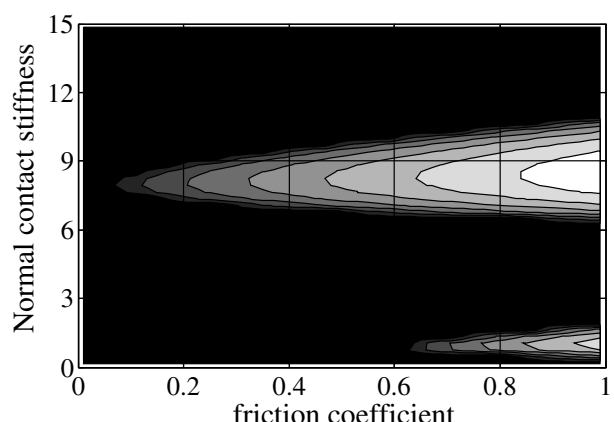

(b)

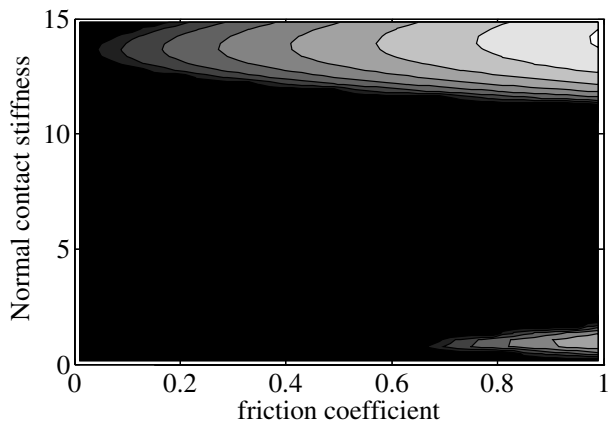

(d)

Fig. 6. Stability region of the system with DVA applied in horizontal direction of: (a) $v=0.05$ and $f=0.2 \mathrm{~Hz}$, (b) $v=0.3$ and $f=0.4 \mathrm{~Hz},(\mathrm{c}) v=0.2$ and $f=0.5 \mathrm{~Hz}$ and (d) $v=0.4$ and $f=0.5 \mathrm{~Hz}$.

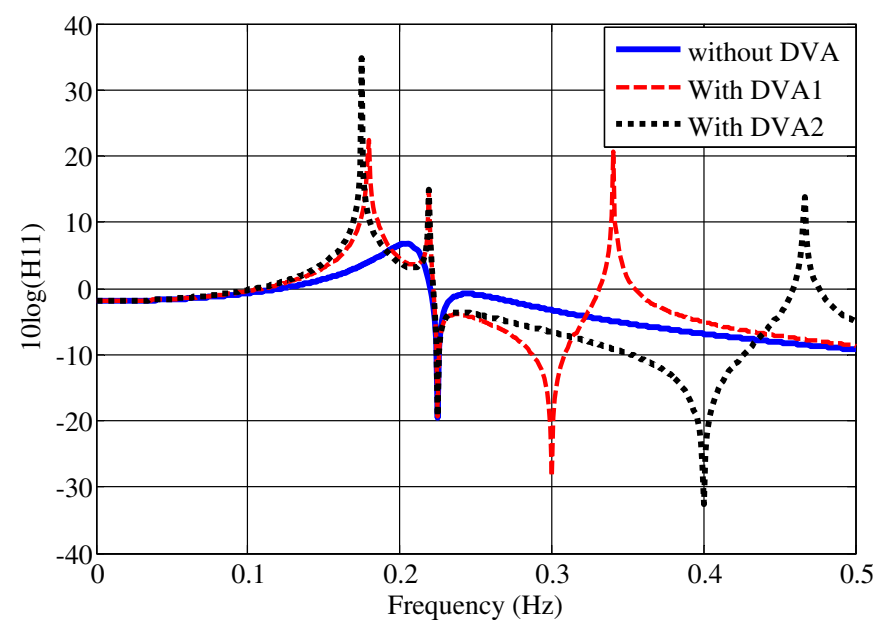

Fig. 7. Frequency response function of the system with DVA. 
DVAs in the horizontal direction, with mass ratio $v=0.2$, DVA frequency $f=0.3 \mathrm{~Hz}$ (DVA1) and $v=0.3, f=0.4 \mathrm{~Hz}$ (DVA2), are able to break the unstable coupling mode into two stable modes.

\section{Multi Degree of Freedom Model}

The second example considered is the three-dimensional finite element model of a simple L-shape frame made of stainless steel with a friction contact at one edge, as shown in Fig. 8. ${ }^{25}$ The entire frame is modeled by nine finite elements with a constant cross sectional area. The friction force is applied only at point 9 along the $x$-axis of the global coordinate system, and the normal contact stiffness is set variously up to $1 \times 10^{8} \mathrm{~N} / \mathrm{m}$. Tangential contact force caused by the tangential contact stiffness is accumulated to the total friction force as represented by the dynamic friction coefficient of 0.5 .

Figure 9 shows the interaction of some eigenvalues versus the normal contact stiffness. Two eigenvalues (6th and 7th modes) produce a mode coupling within the frequency range from $1800 \mathrm{~Hz}$ to $2100 \mathrm{~Hz}$, and within the normal contact stiffness between $1 \times 10^{7}$ and $12 \times 10^{7} \mathrm{~N} / \mathrm{m}$. It is noted here that some natural frequencies in Fig. 9 remain unchanged irrespective of the normal contact stiffness in the

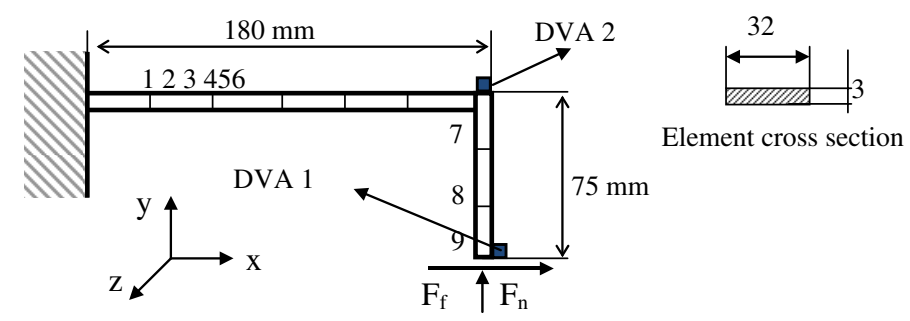

Fig. 8. L-shaped frame model designed to produce squeal noise. ${ }^{25}$

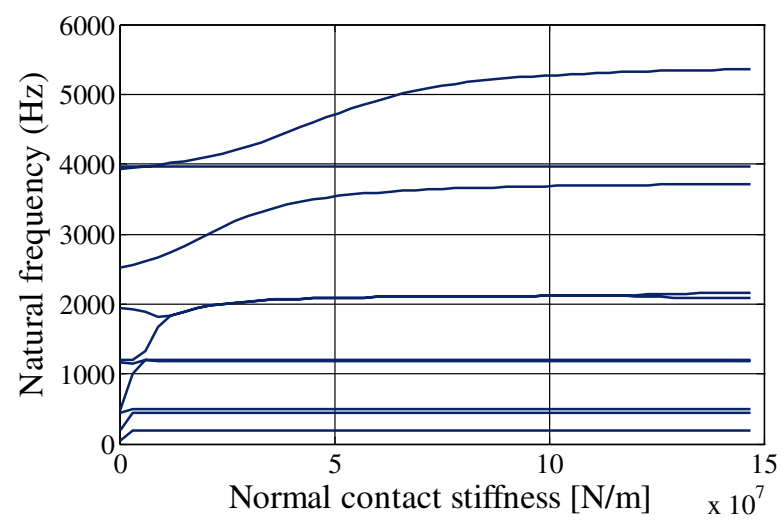

Fig. 9. Natural frequency of L-shape frame versus normal contact stiffness. 

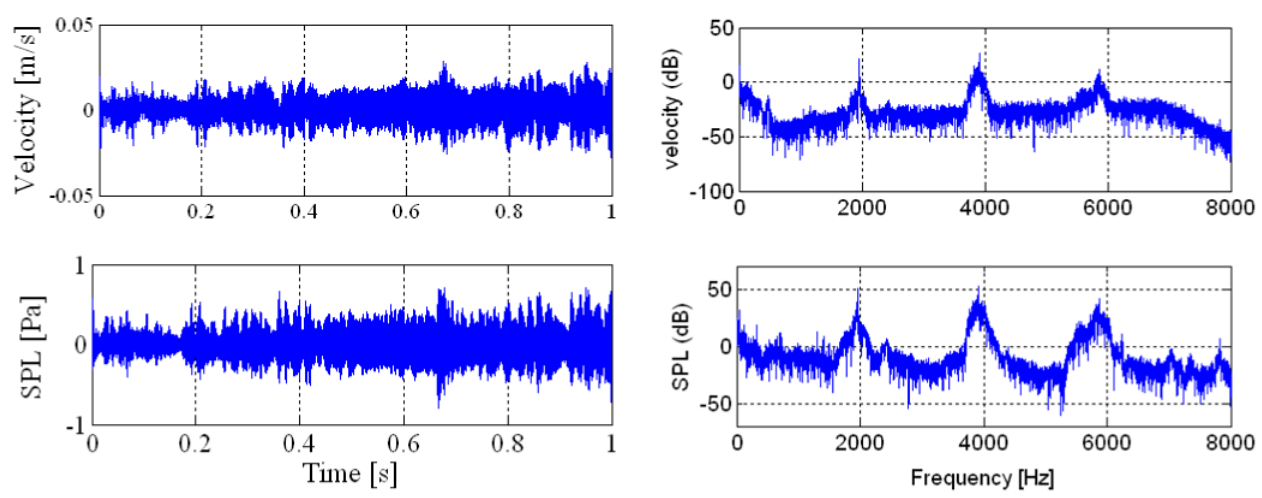

Fig. 10. Vibration response and sound pressure level in squeal noise. ${ }^{25}$

$x$-direction, because some of them are natural modes in the $x z$ or $y z$-planes of the three-dimensional frame model.

In the experimental observation, it was found that when a slider bar was moved into the $x$-direction, and starts scratching point 9 of the L-shape structure, squeal noise was steadily emitted during the sliding. ${ }^{25}$ The vibration response at point 5 and the sound pressure level of the squeal noise are depicted in time domain and frequency domain in Fig. 10. Clearly, the squeal noise consists of only a few spectra at $1955 \mathrm{~Hz}$ (fundamental harmonics), $3910 \mathrm{~Hz}$ (second harmonics), and $5865 \mathrm{~Hz}$ (third harmonics).

Previously, numerical analysis for the TDOF model suggested the idea that an added mass with very stiff spring or an added rigid mass in the horizontal direction may remove unstable mode coupling. In the following, the application of an added mass to remove unstable mode coupling in the L-shape frame model will be shown. First, a particle rigid mass of 2, 5, 7 or 10 grams is added on point 9 of the L-shape frame in (Fig. 8), or at the near-friction-point. In the finite element model, the mass has three translational DOGs. The result is shown in Fig. 11.

Figure 11(a) shows that the two-gram added mass on point 9 changes the mode coupling between modes 6 th and 7 th, restricting it to occur within the normal contact stiffness of $1 \times 10^{7}-3 \times 10^{7} \mathrm{~N} / \mathrm{m}$. Hence, the possibility of unstable mode coupling occurrence has been reduced. Furthermore, as indicated by Fig. 11(b), the 5 -g added mass almost eliminates the possibility of squeal noise by reducing the region of instability to that within the normal contact stiffness of $1 \times 10^{7}-1.3 \times 10^{7} \mathrm{~N} / \mathrm{m}$. Finally, attaching a 7 -g or 10 -g added mass is able to entirely suppress the possibility of squeal noise generation at those modes.

Furthermore, the frequency responses are shown in Fig. 12 for the normal contact stiffness of $2 \times 10^{7} \mathrm{~N} / \mathrm{m}$, and the friction coefficient of 0.5 , in which is located within the unstable region illustrated in Fig. 9. The structure without added mass has an unstable mode coupling at the frequency around $2000 \mathrm{~Hz}$ where the peak spectrum is very low. In previous discussions, it was found that the unstable mode coupling has a 


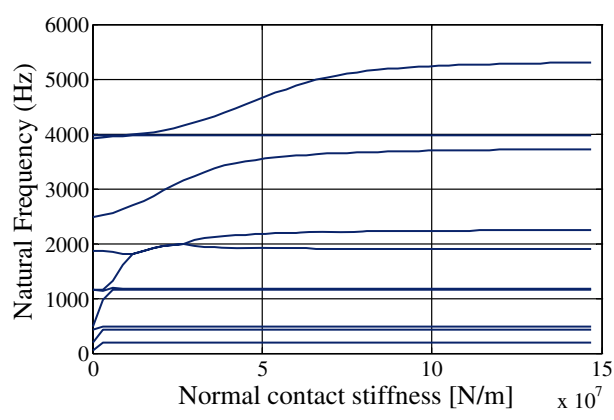

(a)

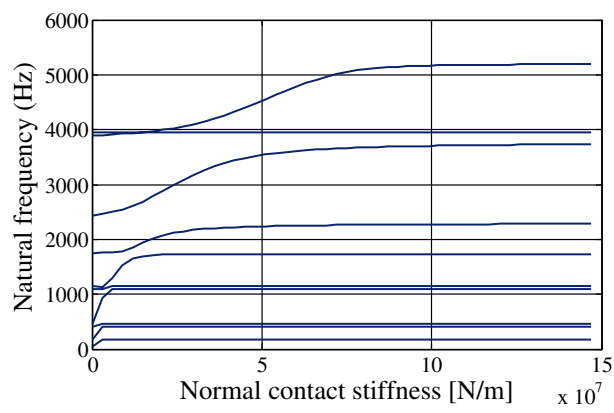

(c)

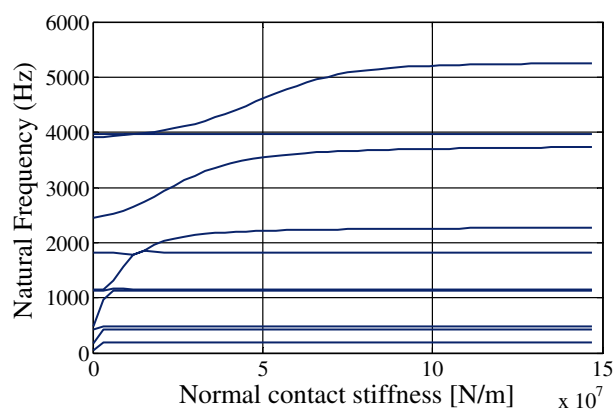

(b)

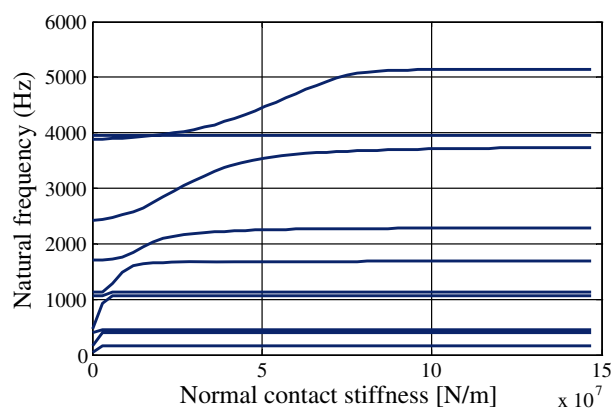

(d)

Fig. 11. Natural frequencies with added mass at point 9 of: (a) $2 \mathrm{~g}$, (b) $5 \mathrm{~g}$, (c) $7 \mathrm{~g}$ and (d) $10 \mathrm{~g}$.

low peak frequency response and a dominant unstable transient response at the mode coupling frequency. This means that not only its coupling mode frequency peak, but other frequency peaks in frequency response spectrum will be dominated by this unstable transient response, too. This may explain why most of squeal noise incident

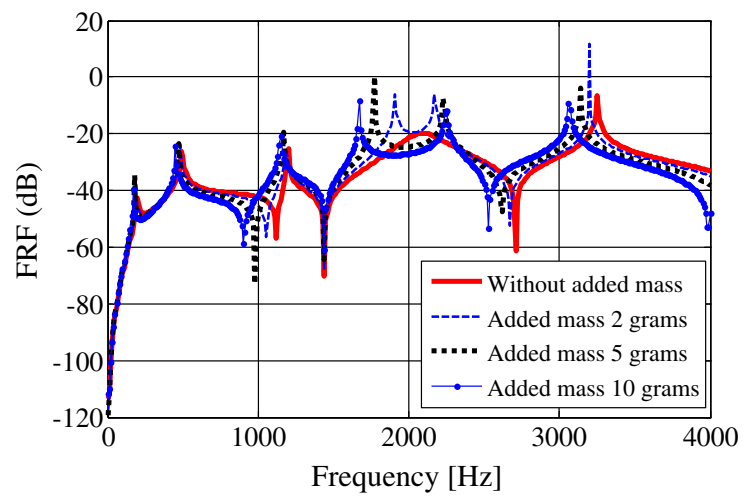

Fig. 12. Frequency response functions with varied added mass at point 9 . 
has only a single peak in the frequency spectrum. Moreover, adding two-gram mass or more has the possibility to break the unstable coupling mode into two stable modes. In particular, it was shown that attaching 10-g mass can effectively prevent instability, while generating lower frequency response on the structure.

In the next, a particle rigid mass is attached to point 6 of the L-shape frame. The added mass is allowed to vary from 2 grams, 5 grams, 10 grams, to 15 grams. In Fig. 13(a), it was shown that by adding a two-gram mass on point 6 , mode coupling between modes 6 and 7 th still occur at within frequency of 1800-2000 Hz and wider range of normal contact stiffness of $1 \times 10^{7}-12.5 \times 10^{7} \mathrm{~N} / \mathrm{m}$. The possibility of occurrence of unstable mode coupling to generate squeal noise is increased. Also, the addition of bigger masses (5, 10 and 15 g), as shown in Figs. 13(b)-13(d), raises the possibility to generate the unstable mode coupling. The frequency responses are shown in Fig. 14 for the normal contact stiffness of $2 \times 10^{7} \mathrm{~N} / \mathrm{m}$, and friction coefficient of 0.5 , which is located within the unstable region shown in Fig. 9. It is confirmed that added mass up to $15 \mathrm{~g}$ on point 6 is unable to separate unstable coupling mode into two stable modes. In other words, it is ineffective prevent instability of the structure by this mechanism. Therefore, it is concluded that the mass addition on point 9 is effective to reduce the possibility of squeal noise incident; however, it is unsuccessful when attached on point 6 .

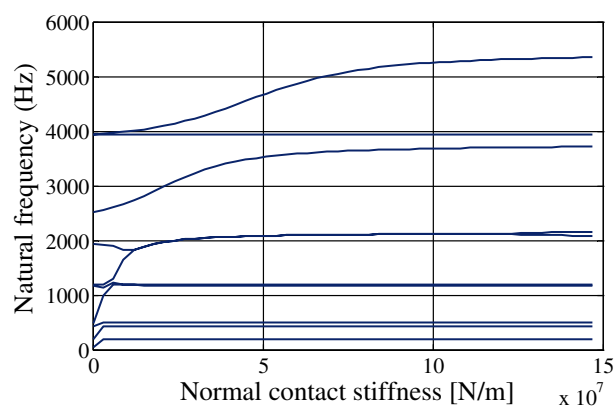

(a)

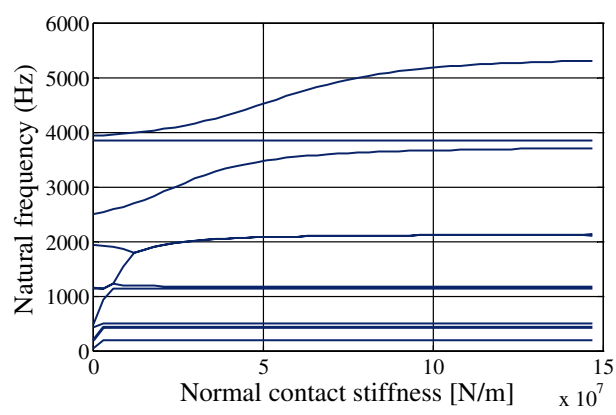

(c)

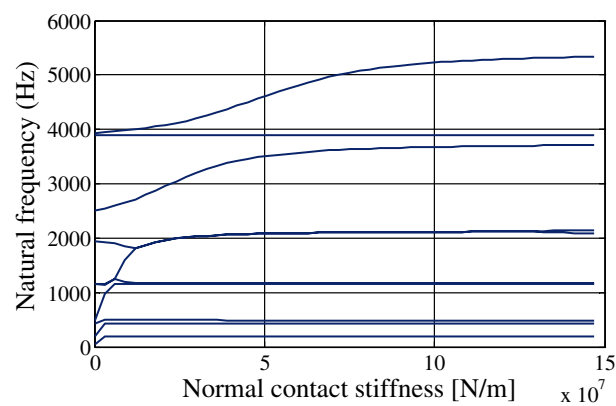

(b)

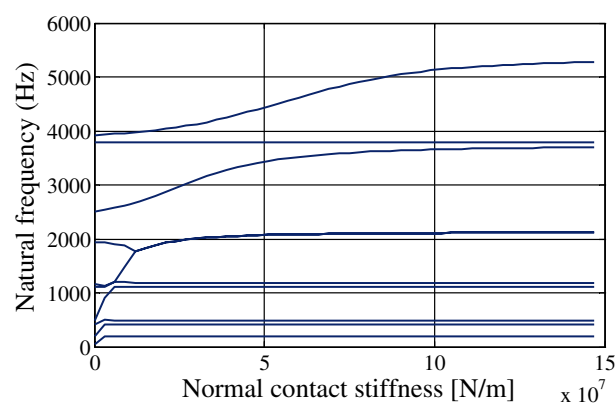

(d)

Fig. 13. Natural frequencies with added mass at point 6 of: (a) $2 \mathrm{~g}$, (b) $5 \mathrm{~g}$, (c) $10 \mathrm{~g}$ and (d) $5 \mathrm{~g}$. 


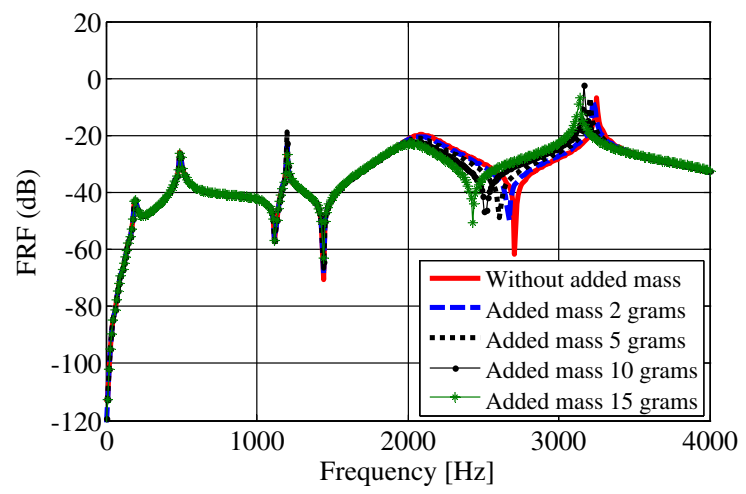

Fig. 14. Frequency response function with varied added mass at point 6 .

Based on the investigation by Hoffman, ${ }^{24}$ it was found that energy can be transferred from the frictional to the vibratory system due to a simple mechanism: the normal force oscillations can lead to oscillations of the tangential frictional force. If there are simultaneously relative tangential displacements at the friction interface that are not exactly in phase with the force oscillations, a cyclic growth of the vibrational energy may occur. Also, the friction force acts like a structural coupling force linking the out-of-plane motion to in-plane motion and that instability may occur if these friction-induced cross-coupling forces balance the corresponding coupling forces of the structural system.

The addition of a mass-spring system in the vertical direction will increase the normal force oscillation, leading to oscillation of the tangential frictional force. In the TDOF model, it was observed that the added mass in the vertical direction increases the possibility of squeal noise incident. Similarly, the added mass on point 6 of the Lshape structure increases the possibility of instability occurrence. The added mass on point 6 moves flexibly in the vertical direction but very difficult in the horizontal direction, because of the structural rigidity. Therefore, the added mass leads to increase of the normal force oscillation.

On the other hand, the added mass in horizontal direction at the near-point-offriction absorbs more energy to move the structure and added mass. In the case of the TDOF model, it is observed that adding a bigger mass in horizontal direction shifts the possibility of squeal noise incident to higher normal contact stiffness. It means that more energy is needed to make the system unstable. Similar to the TDOF model, the added mass on point 9 reduces the possibility of instability occurrence. The added mass on point 9 moves flexibly in the horizontal direction. The 6 th and 7th mode shapes that generate a coupling mode have high eigenvectors to the horizontal direction in point 9, as shown in Fig. 15. Higher kinetic energy is needed to move the structure to follow the mode shape. Therefore, higher friction forces or higher friction coefficients are needed to link the out-of-plane motion to in-plane motion. 

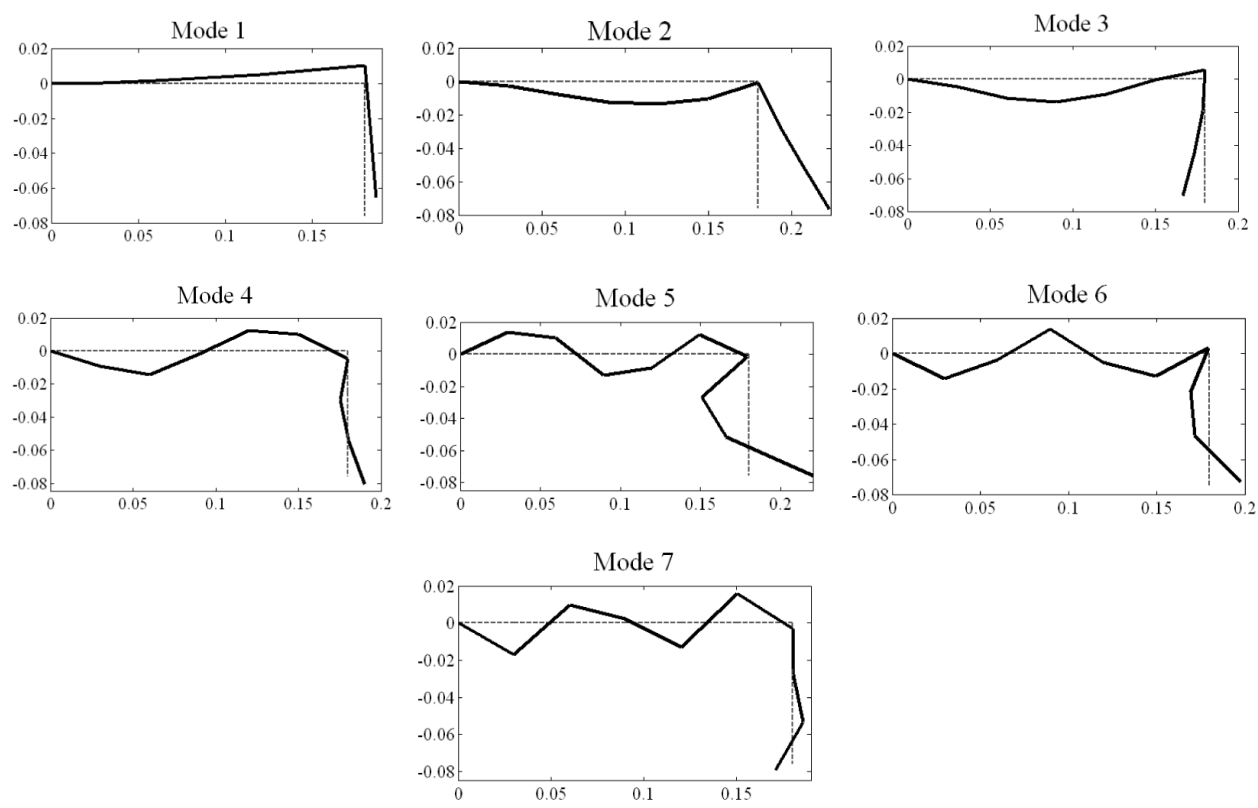

Fig. 15. First seven mode shapes. ${ }^{25}$

In addition, the mechanism of energy transfer and bifurcation point of mode coupling system in the friction induced vibration and noise is still not clearly explained. It should be mentioned that the instability of the structure considered is due to nonsymmetric stiffness matrices. Mathematically, any nonsymmetric matrix can be decomposed into a symmetric and a skew-symmetric; however, in the case of friction self-induced vibration, this decomposition can hardly be given any physical meaning.

\section{Conclusion}

The application of DVAs in suppressing squeal noise has been investigated. The DVA in squeal noise reduction works in different ways compared with a general vibration system. The application of DVAs in general vibration system is aimed to reduce the vibration amplitude of the main system (damped system). However, in the squeal noise reduction, The DVA is implemented to prevent squeal noise caused by mode-coupling instability by shifting or reducing the unstable region, such that the unstable region is removed from the operating condition.

The performance of the DVA is investigated in two simple cases: the simplest TDOF model and the L-shape frame. It was shown that by adding a mass-spring system in vertical direction will increase the possibility of squeal noise incident. In contrast, the addition in horizontal direction at the near-point-of-friction has the possibility to avoid unstable mode coupling by moving the unstable region to one with higher normal contact stiffness. Therefore, an added mass with very stiff spring 
or rigid mass will prevent the occurrence of unstable mode coupling as long as it is uncoupled from the vertical direction. If the added mass is involved in the dynamic responses of both vertical and horizontal directions, the squeal noise with the original normal contact stiffness can still occur.

\section{Acknowledgments}

The authors gratefully acknowledge the financial support from Engineering Faculty, Andalas University by contract number 003/PL/SPK/PNP/FT-Unand/2013. We would like to express profound gratitude to colleagues in Structural Dynamics Laboratory and Department of Mechanical Engineering for the guidance, help, and cooperation, and to colleagues from American corner Andalas University for checking the English grammar of this paper.

\section{References}

1. R. A. Ibrahim, Friction-induced vibration, chatter, squeal, and chaos - Part II: Dynamics and modeling, ASME Appl. Mech. Rev. 47 (1994) 227-253.

2. C. J. M. Van Ruiten, Mechanism of squeal noise generated by trams, J. Sound Vibr. 120 (1988) 245-253.

3. M. A. Heckl and I. I. Abrahams, Curve squeal of train wheels, Part I: Mathematical model for its generations, J. Sound Vibr. 229 (2000) 669-693.

4. F. G. de Beer, M. H. A. Janssens and P. P. Kooijman, Squeal noise of rail-bound vehicles influenced by lateral contact position, J. Sound Vibr. 267 (2003) 497-507.

5. N. M. Kinkaid, O. M. O'Reilly and P. Papadopoulos, Review automotive disc brake squeal, J. Sound Vibr. 267 (2003) 105-166.

6. O. Ouyang, J. E. Mottershead and W. Li, A moving-load model for disc brake stability analysis, Trans. ASME J. Vibr. Acoust. 125 (2003) 53-58.

7. M. Eriksson, F. Bergman and S. Jacobson, Surface characterization of brake pads after running under silent and squealing condition, Wear 232 (1999) 163-167.

8. F. Bergman, M. Eriksson and S. Jacobson, Influence of disc topography on the generation of brake squeal, Wear 225-229 (1999) 621-628.

9. P. J. Remington, Wheel/rail squeal and impact noise: What do we know? What don't we know? Where do we go from here, J. Sound Vibr. 116(2) (1986) 339-353.

10. D. T. Eadie, M. Santoro and J. Kalousej, Railway noise and the effect of top of rail liquid friction modifiers: Changes in sound and vibration spectral distributions in curves, Wear 258 (2005) 1148-1155.

11. J. F. Brunel, P. Dufrenoy and F. Demily, Modeling of squeal noise attenuation of ring damped wheels, Appl. Acoust. 65 (2004) 457-471.

12. M. A. Botto, J. M. C. Sousa and J. M. G. Sá da Costa, Intelligent active noise control applied to a laboratory railway coach model, Control Eng. Pract. 13 (2005) 473-484.

13. R. A. C. Fosberry and Z. Holubecki, Some experiments on the prevention of brake squeal, MIRA Technical Report, No. 1957/1 (1957).

14. T. Hamabe, I. Yamazaki, K. Yamada, H. Matsui, S. Nakagawa and M. Kawamura, Study of a method for reducing drum brake squeal, SAE Technical Paper 1999-01-0144 (1999), pp. $53-60$.

15. K. A. Cunefare and A. J. Graf, Experimental active control of automotive disc brake rotor squeal using dither, J. Sound Vibr. 250 (2002) 579-590. 
16. J. P. Den Hartog, Mechanical Vibration (McGraw-Hill, New York, 1947).

17. L. Zuo and S. A. Nayfeh, Minimax optimization of multi-degree-of-freedom tuned-mass dampers, J. Sound Vibr. 272 (2004) 893-908.

18. S. V. Bakre and R. S. Jangid, Optimum multiple tuned mass dampers for base-excited damped main system, Int. J. Struct. Stability Dynam. 4(4) (2004) 527-542.

19. B. Khan and C. X. Li, Evaluation of multiple dual tuned mass dampers for structures under harmonic ground acceleration, Int. J. Struct. Stability Dynam. 6(1) (2006) 59-75.

20. M. H. Miguelez, L. Rubio, J. A. Loya and J. Fernandez-Saez, Improvement of chatter stability in boring operations with passive vibration absorbers, Int. J. Mech. Sci. 52 (2010) 1376-1384.

21. Sunny K. George and K. Shankar, Vibrational energies of members in structural networks fitted with tuned vibration absorbers, Int. J. Struct. Stability Dynam. 6(2) (2006) 269284.

22. A. R. Abu-Bakar, M. R. Buang and M. Z. A. Rashid, Suppression of drum brake squeal through structural modifications using finite element method, Int. J. Vehicle Design $\mathbf{5 1}$ $(1 / 2)(2009) 3-20$.

23. J. Sinou and L. Jezequel, Mode coupling instability in friction-induced vibrations and its dependency on system parameters including damping, Eur. J. Mech. - A/Solids 26(1) (2007) 106-122.

24. N. Hoffman, M. Fischer, R. Allgaier and L. Gaul, A minimal model for studying properties of the mode-coupling type instability in friction induced oscillation, Mech. Res. Commun. 29 (2002) 197-205.

25. M. Rusli and M. Okuma, Squeal noise prediction in dry contact sliding systems by means of experimental spatial matrix identification, J. Syst. Design Dynam. 2(3) (2008) $585-595$.

26. A. P. Seyranian and A. A. Mailybaev, Interaction of eigenvalues in multi-parameter problems, J. Sound Vibr. 267 (2003) 1047-1064.

27. N. Hoffman and L. Gaul, Effect of damping on mode-coupling instability in friction induced oscillations, ZAMM Z. Angew. Math. Mech. 83(8) (2003) 524-534.

28. M. Rusli and M. Okuma, Effect of surface topography on mode-coupling model of dry contact sliding systems, J. Sound Vibr. 308 (2007) 721-734.

29. H. A. Sherif, Mode of zero wear in mechanical systems with dry contact, Tribol. Int. 38 (2005) 59-68. 